\title{
Navigation and Object Detection for Blind Persons Based on Neural Network
}

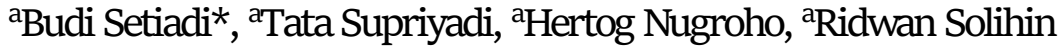 \\ aDepartment of Electrical Engineering, Bandung of State Polytechnic, Bandung 40012, Indonesia
}

Received 14 October 2019; accepted 19 February 2020

\begin{abstract}
Tools for blind people with mobility activities in pedestrian pathways have been widely launched, approved and patented. However, there are still shortcomings that can be done only for pedestrian paths or nearby destinations. In this study, both a camera (detection of the pedestrian path) and LiDAR (detection of surrounding objects) sensors to help disability activities. The first stage of image data from the preparatory camera from RGB to XYZ, color filters, close morphology, resizing, learning and testing of neural networks. Bring up 3 voice attitudes information. Attitudes are perpendicular, left tilted, right tilted, or not reversed to the pedestrian yellow path. The second stage of the LiDAR distance points data is processed into $2 \mathrm{D}$ array geometry, learning, and testing of neural networks. Bring up the information 8 voice attitudes. Detection of the cycle and distance of objects right side, front, left, right-front, right-left, front-left, right-front-left, not captured. The test results approximately at lux $<15000$ got $89.7 \%$ accuracy for pedestrian path detection and $87.5 \%$ for object detection.
\end{abstract}

\section{KEYWORDS}

Blind persons

Neural network

LiDAR

\section{INTRODUCTION}

Products, patents, and research that discusses discussing a cane (white can) and the like to help blind persons with disabilities along the pedestrian lane have been carried out. Either a combination of assistive devices with sensors or using a pure camera. The sensors used are ultrasonic, infrared (IR), Accelero \& PIT, RFID, 3D TOF to detect surrounding objects. Ultrasonic function is to detect objects of inanimate objects around them with a range of a distance of 2 meters. Infra-red functions to detect objects of living objects around them. Accelero \& PIT serves to detect the direction of motion and in the event of rotation, calculated from the time of change in the $\mathrm{X}$ and $\mathrm{Y}$ axis values. All data information issued in the form of different tones to the buzzer (Al Kandari et al., 2016; Sukhdeep et al., 2016, Ganguli et al., 2016). Other studies add the GSMGPS Modem function to inform the blind position of blind persons automatically or manually through a button if an incident occurs (Dhananjeyan et al., 2016).

Other recognition techniques use cameras to work along the pedestrian lane. Detect pedestrian lines that are marked with two white lines at a traffic junction. Uses extra ROIverification of lanes and lanes. Color and intensity information to detect lane markers, and then 


\section{BUDI S. ET.AL}

verify probabilistic work candidate areas. The geometric feature of the marker lane is used for verification. Pedestrian lane detection in an unstructured environment. Integrate both the appearance of the region and the characteristics of the border lane (Le et al., 2012; Mocanu et al., 2018). The vanishing point is used to detect lane boundaries based on the color edge orientation, and to use pedestrian detection for occlusion handling. The pedestrian lane detection method is evaluated on a new data set of 2000. And the object recognition technology uses a camera with the Convolutional Neural Network method (Le et al., 2014).

This research proposes a combination of combining two sensors working together and complementing each other, as well as a new algorithm converting juring arrays to $2 \mathrm{D}$ arrays. When the camera sensor and LiDAR produce good data, the 3 attitudes of sound information (object navigation from the camera, as well as detection, object distance, and spatial geometry from LiDAR) will be generated. When the data from the camera sensor is not good, then the attitude of the sound information is generated from LiDAR only and vice versa.

\section{RESEARCH METHOD}

The data processing stages proposed are of two types, namely navigation and detection of surrounding objects on the pedestrian lane. The navigation stage of the data input is an RGB image captured by the camera. The object detection stage of data input is a $2 \mathrm{D}$ array by LiDAR catches. The following is an explanation of the hardware block diagram, shown in Figure 1.

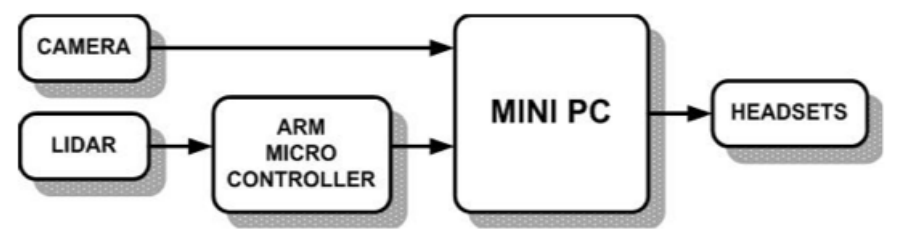

\section{Navigation Process from Camera}

The navigation steps of pedestrian lane detection are processed on the mini PC, the following sequence is as follows, shown in Figure 2. 


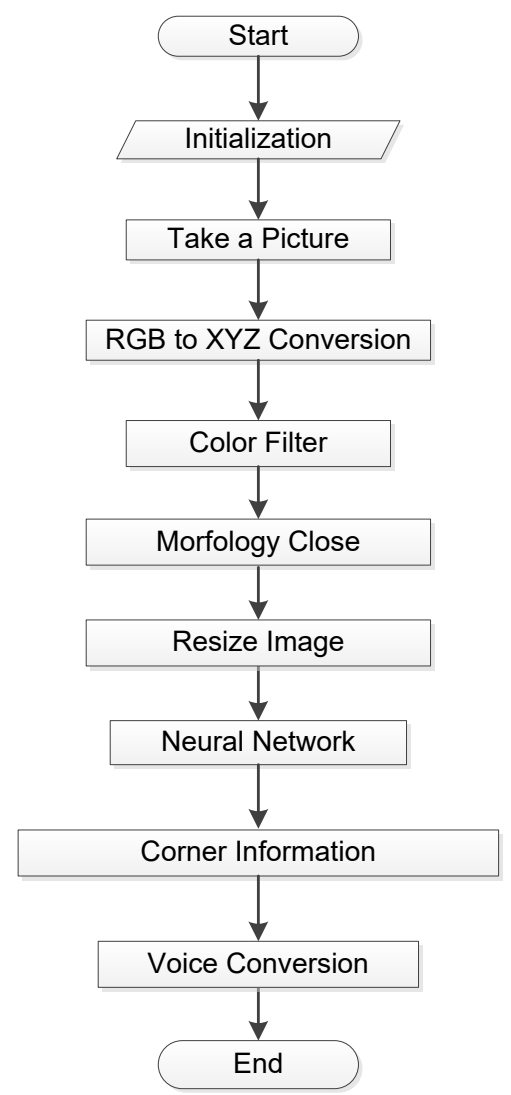

Figure 2. Navigation Flow Chart

The navigation steps of the pedestrian lane detection are processed a) Input the RGB standard RGB color image array captured by the camera is converted to the XYZ color system. Meanwhile, the conversion of RGB to XYZ colors is shown in equation 1.

a) The navigation steps of the pedestrian lane detection are processed a) Input the RGB standard RGB color image array captured by the camera is converted to the XYZ color system. Meanwhile, the conversion of RGB to XYZ colors is shown in equation 1.

$$
\left[\begin{array}{l}
X \\
Y \\
Z
\end{array}\right]=\frac{1}{0.176,97}\left[\begin{array}{llll}
0.49000 & 0.31000 & 0.200 & 00 \\
0.17697 & 0.81240 & 0.01063 \\
0.00000 & 0.01000 & 0.99000
\end{array}\right]\left[\begin{array}{l}
R \\
G \\
B
\end{array}\right]
$$

b) The color filter can isolate the pedestrian lane from other objects, so it is expected that from the drawing the lane is left only; Meanwhile, the screening process made specifically for this case, is shown by the press equation 2 . 
BUDI S. ET.AL

$$
b w=\left\{\begin{array}{cc} 
& x_{l}<X<x_{u} \\
1 & y_{l}<Y<y_{u} \\
& z_{l}<Z<z_{u} \\
0 & \text { else }
\end{array}\right.
$$

c) Close morphology to highlight the pedestrian lane as much as possible. The chosen process is morphology 'close', where s is a structured element. Meanwhile, the results are shown by the press equation 3 .

$$
B W \cdot s=\left(B W \bigoplus_{\text {Srot }}\right) \ominus_{\text {Srot }}
$$

d) Resize image to the 500x500 pixel neural network architecture input layer. The core changes size by assuming colors between two pixels that are known to be linear. In this model, the color of a pixel is determined by the four pixels that surround it.

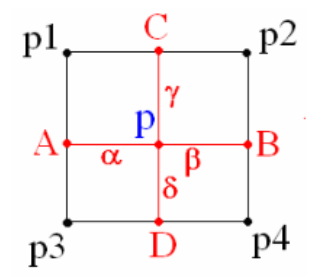

Figure 3. Resize Image

Resize image adjust to the input layer of neural network architecture. The core changes size by assuming colors between two pixels that are known to be linear. In this model, the color of a pixel is determined by the four pixels that surround it. Points p1, p2, p3, and p4 are the pixels whose colors are known while $\mathrm{p}$ is the pixels to be searched for by the bilinear interpolation approach.

\begin{tabular}{|c|c|c|c|}
\hline \multirow{2}{*}{ No. } & \multirow{2}{*}{ Input Image Data } & \multicolumn{2}{|c|}{ Output Target } \\
\hline & & $\mathrm{t} 1$ & t2 \\
\hline 1 & Data lane yellow sloping left side & $\mathrm{O}$ & $\mathrm{O}$ \\
\hline 2 & Data lane yellow sloping right side & $\mathrm{O}$ & 1 \\
\hline 3 & The data lane is perpendicular to yellow & 1 & $\mathrm{O}$ \\
\hline 4 & Pedestrian lane not detected & 1 & 1 \\
\hline
\end{tabular}

Table 1. Camera Learning Pattern

From the learning results generated $500 \times 500$ new weights and 1 new bias.

f) Do a test using new weights and bias in learning outcomes. Testing is done directly from the camera on a mini PC device that has been planted with the results of the research algorithm. 


\section{Object Detection from LiDAR}

The object detection steps of pedestrian lane detection are processed on the ARM Microcontroller, the following sequence is as follows, shown in Figure 4.

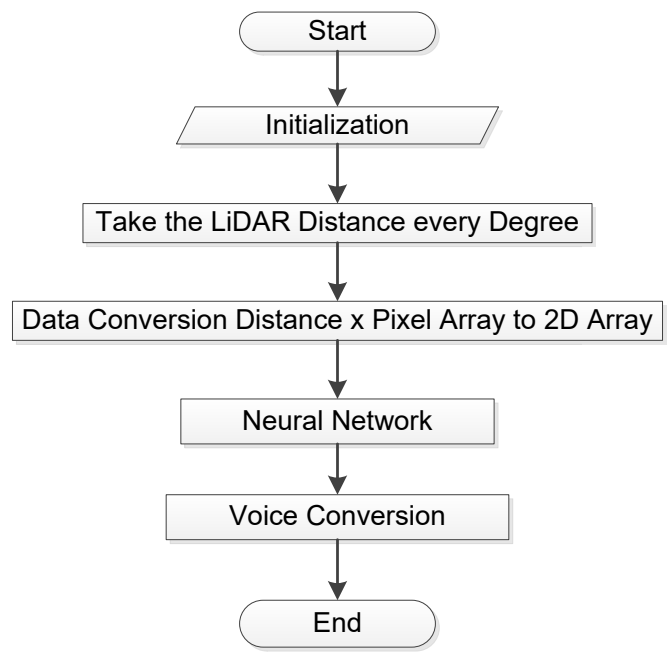

Figure 4. Object Detection Flow Chart

a) LiDAR data capture in the form of pixel spacing every $1^{\circ}$ with one packet sending amounting to $6^{\circ}$ with 42 Byte format. Rotating $360^{\circ}$ automatically horizontally in a clockwise direction and vertical 100 steps produce an array of data scaling. Design considerations for horizontal prototype pixels are limited to $196^{\circ}$ and measurement distances from $100 \mathrm{~mm}$ to $2000 \mathrm{~mm}$.

b) b) Convert captured arrays of juring arrays which are still in the form of distance pixels to $2 \mathrm{D}$ array pixels.
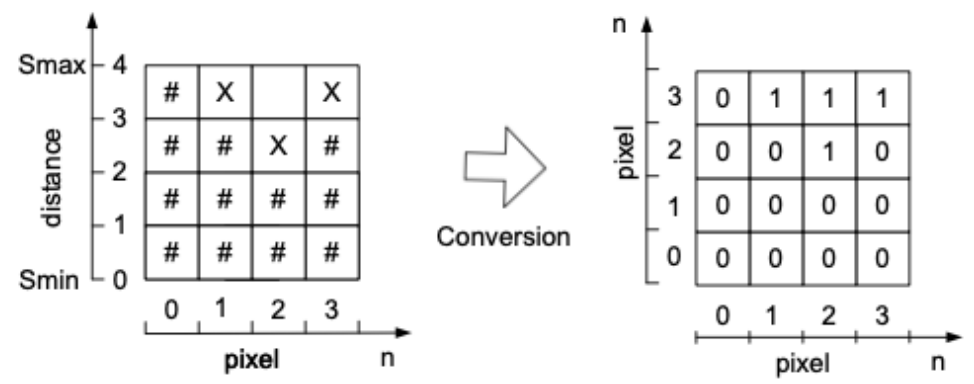

Figure 5. Algorithm Juring Array to 2D Conversion

Algorithm array conversion to 2D, as follows:

1. Determine the number of distance pixels;

2. Number of pixels distance $=$ number of pixels (n);

3. The dimensions of the $2 \mathrm{D}$ array conversion result are $\mathrm{n} x \mathrm{n}$ pixels;

4. Smax-the distance measurement result, if the result is $\mathrm{O}$, then fill the pixel distance from Smin to Smax with the value o; 
BUDI S. ET.AL

5. Smax-measurement distance results, if the result is $>0$, then fill in the pixel distance from Smin to the measurement distance result with a value of $\mathrm{O}$ and from the measurement distance result to the Smax value of 1 .

c) Learn from the sample data using the help of Matlab to obtain new weights and biases values. System architecture uses the backpropagation method. Consists of the input layer (196 x 196 neurons), hidden layer 20 neurons, and output layer 3 neurons.

Table 2. LiDAR Learning Pattern

\begin{tabular}{clccc}
\hline \multirow{2}{*}{ No. } & \multicolumn{2}{c}{ Input Data Position Pattern } & \multicolumn{3}{c}{ Output Target } \\
\cline { 3 - 5 } & & t1 & t2 & t3 \\
\hline 1 & Right object position data & 0 & o & O \\
2 & Front object position data & 0 & 0 & 1 \\
3 & Left object position data & 0 & 1 & 0 \\
4 & Position data of right-front objects & 0 & 1 & 1 \\
5 & Left-front object position data & 1 & 0 & 0 \\
6 & Position data of right-left objects & 1 & 0 & 1 \\
7 & Position-right-left-left object data & 1 & 1 & 0 \\
8 & There are no objects & 1 & 1 & 1 \\
\hline
\end{tabular}

From the learning results produced 196 x 196 new weights and 1 new bias values.

d) Do a test using new weights and bias in learning outcomes. Testing is done directly from LiDAR.

e) The array data is searched for the minimum distance value using the algorithm and.

\section{Voice Information Attitude}

Information stages 3 voice attitudes are processed on the mini PC for the input camera sensor. And Information stages 8 voice attitudes are processed on the mini PC for input LiDAR sensor. Each numerical value of attitude output in sequence is compared one by one with a sound database on the mini PC. Then transmitted via the Bluetooth module to the headset.

\section{RESULT AND ANALYSIS}

\section{Product Design}

The research output is in the form of a waist bag prototype, as shown in Figure 6. The detailed function of the components used is as follows: camera (1), LiDAR (2), mini PC (3), microcontroller ARM (4), array 2D (5), array juring (6), bags (7), headset (8). 


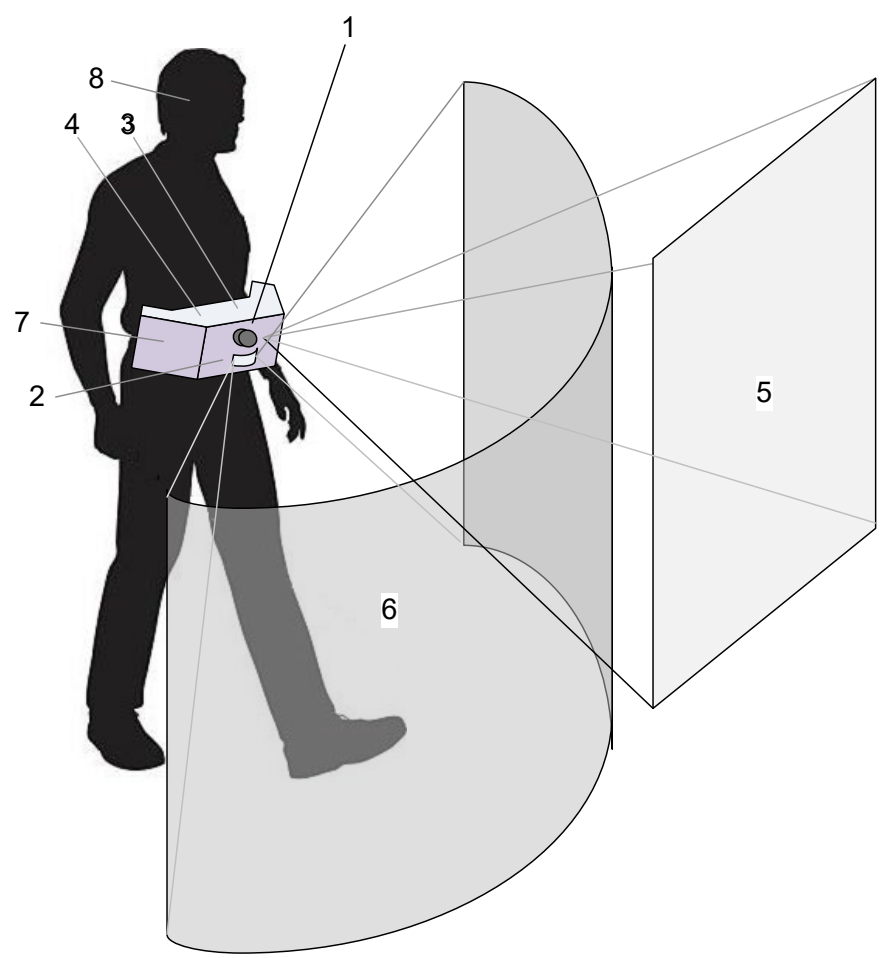

Figure 6. Prototype

\section{Camera Testing}

Learning data consists of 400 sample images. Sample images were taken at 4 different locations, namely PSBN Wiyata Guna Bandung, Cicendo, Asia Afrika, and Tamansari Street, shown in Table 3. the test is carried out in the same place and condition as the learning sample. testing is done 400 times. 100 tests each at each location, shown in Table 4.

Table 3. Data Sample Learning camera

\begin{tabular}{cllc}
\hline No. & \multicolumn{1}{c}{ Location } & Pedestrian Data Type & Number of Samples \\
\hline 1 & PSBNWiyata Guna & Without Object & 100 \\
2 & Cicendo Street & Without Object & 100 \\
3 & Asia Afrika Street & Without Object & 100 \\
4 & Tamansari Street & Without Object & 100 \\
\hline
\end{tabular}

Table 4. Data Testing camera

\begin{tabular}{|c|c|c|c|c|c|c|c|}
\hline No. & Location & Testing & $\begin{array}{c}\text { Number of } \\
\text { Testing }\end{array}$ & $\begin{array}{l}t_{1}=0 \\
t_{2}=0\end{array}$ & $\begin{array}{l}t_{1}=0 \\
t_{2}=1\end{array}$ & $\begin{array}{l}t_{1}=1 \\
t_{2}=0\end{array}$ & $\begin{array}{l}t_{1}=1 \\
t_{2}=1\end{array}$ \\
\hline 1 & PSBN Wiyata Guna & Without Object & 100 & 28 & 32 & 29 & 11 \\
\hline 2 & Cicendo Street & Without Object & 100 & 27 & 30 & 29 & 14 \\
\hline 3 & Asia Afrika Street & Without Object & 100 & 30 & 29 & 32 & 9 \\
\hline 4 & Tamansari Street & Without Object & 100 & 30 & 31 & 32 & 7 \\
\hline
\end{tabular}




\section{BUDI S. ET.AL}

Yellow line navigation testing on pedestrian was carried out directly in several points of the city of Bandung. Data collection and testing is carried out during sunny weather, a sample image of data processing results show in Figure 7.
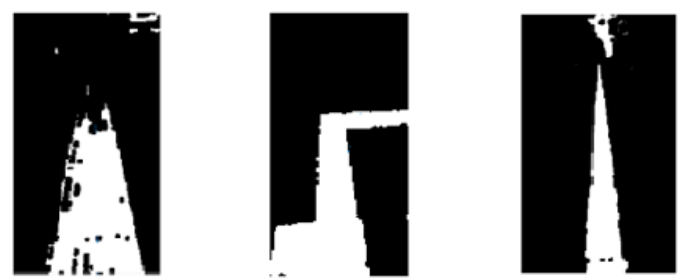

Figure 7. Sample Navigation Using Camera

\section{LiDAR Testing}

Data sample, place and condition the same as camera testing. however, put an object in front of it, shown in Tables 5 and 6.

Table 5. Data Sample Learning LiDAR

\begin{tabular}{clcc}
\hline No. & \multicolumn{1}{c}{ Location } & Pedestrian Data Type & Number of Samples \\
\hline 1 & PSBNWiyata Guna & With Object & 100 \\
2 & Cicendo Street & With Object & 100 \\
3 & Asia Afrika Street & With Object & 100 \\
4 & Tamansari Street & With Object & 100 \\
\hline
\end{tabular}

Table 6. Data Testing LiDAR

\begin{tabular}{|c|c|c|c|c|c|c|c|c|c|c|c|}
\hline No. & Location & Testing & $\begin{array}{l}\text { Number of } \\
\text { Testing }\end{array}$ & $\begin{array}{l}t_{1}=0 \\
t_{2}=0 \\
t_{3}=0\end{array}$ & $\begin{array}{l}t_{1}=0 \\
t_{2}=0 \\
t_{3}=1\end{array}$ & $\begin{array}{l}t_{1}=0 \\
t_{2}=1 \\
t_{3}=0\end{array}$ & $\begin{array}{l}t_{1}=0 \\
t_{2}=1 \\
t_{3}=1\end{array}$ & $\begin{aligned} t_{1} & =1 \\
t_{2} & =0 \\
t_{3} & =0\end{aligned}$ & $\begin{array}{l}t_{1}=1 \\
t_{2}=0 \\
t_{3}=1\end{array}$ & $\begin{array}{l}t_{1}=1 \\
t_{2}=1 \\
t_{3}=0\end{array}$ & $\begin{array}{l}t_{1}=1 \\
t_{2}=1 \\
t_{3}=1\end{array}$ \\
\hline 1 & $\begin{array}{l}\text { PSBN Wiyata } \\
\text { Guna }\end{array}$ & Without & 100 & 13 & 12 & 13 & 11 & 13 & 14 & 14 & 10 \\
\hline 2 & $\begin{array}{l}\text { Cicendo } \\
\text { Street }\end{array}$ & Without & 100 & 12 & 11 & 13 & 13 & 12 & 13 & 13 & 13 \\
\hline 3 & $\begin{array}{l}\text { Asia Afrika } \\
\text { Street }\end{array}$ & Without & 100 & 13 & 12 & 12 & 12 & 14 & 12 & 14 & 11 \\
\hline 4 & $\begin{array}{l}\text { Tamansari } \\
\text { Street }\end{array}$ & Without & 100 & 11 & 12 & 13 & 13 & 12 & 11 & 12 & 16 \\
\hline
\end{tabular}

The object detection test on the pedestrian path is done in the same place as the camera test. Data collection and testing is carried out during sunny weather with trash bin objects, sample images from the results of data processing are shown in Figure 8 and 9. 


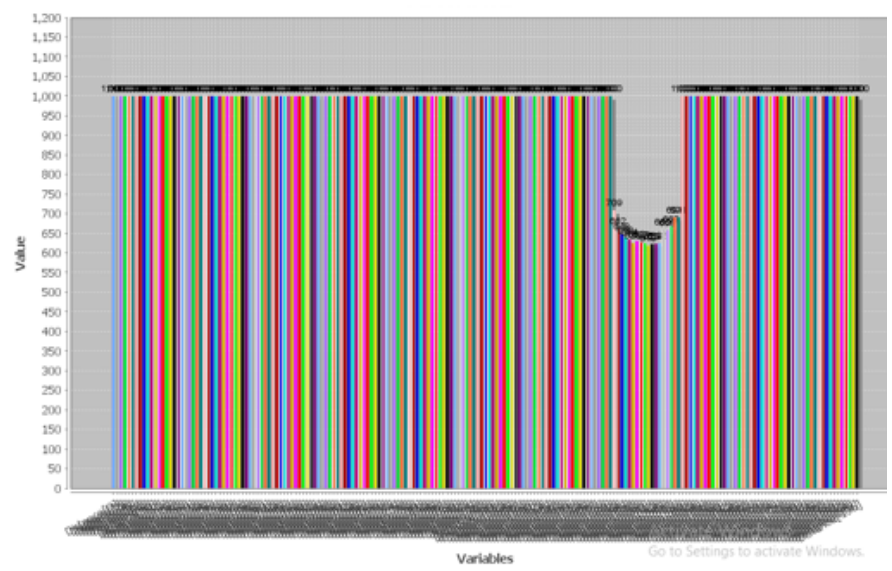

Figure 8. Sample Object Detection, the closest right side distance

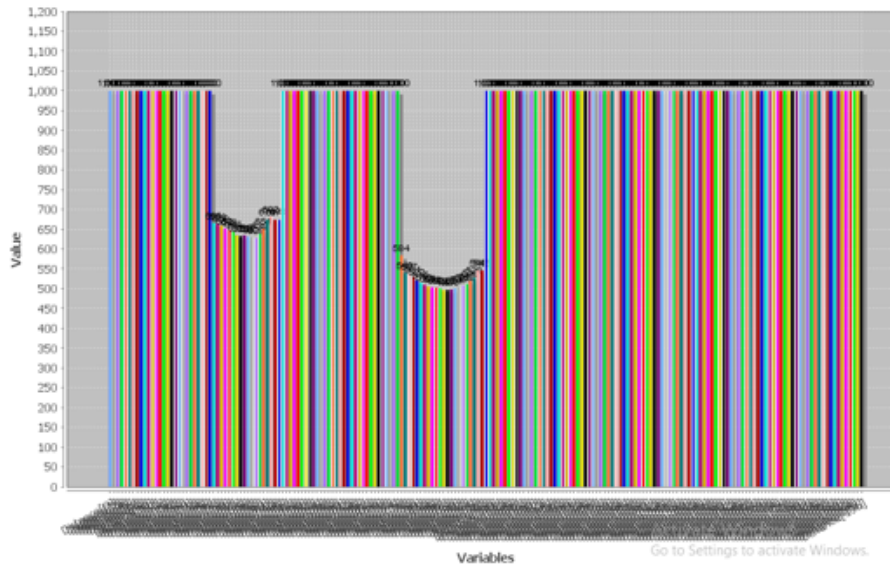

Figure 9. Object Detection, the closest distance to the left and front

\section{CONCLUSION}

The use of camera sensors is interrupted due to the effect of light intensity and vibration. While the LiDAR sensor is disturbed due to the influence of the object's reflection material and the angular bias width. Based on the results of testing and analysis of the use of neural network algorithms Attitude detection of object position, and the closest distance. The measurement test results at lux $<15000$ got $89.7 \%$ success accuracy for pedestrian path detection and 87.5 for object detection.

\section{REFERENCES}

Al Kandari, A., Alajmi, A., Muhammad, S., Alshammari. 2016. Ultrasonic sensors gloves for blind people using Lilypad Arduino. International Journal of New Computer Architectures and their Applications (IJNCAA), 6(1)16-6(1)22.

Andhare, S., Pise, A., Gopanpale, S. 2016. Automated Mobility and Orientation System for Blind. International Research Journal of Engineering and Technology (IRJET), 1762-1765. 
Dhananjeyan, S., Sundaram, K., Kalaiyarasi, A., Kuppusamy, P. 2016. Design and Development of Blind Navigation System using GSM and RFID Technology. Indian Journal of Science and Technology, $9(2) 1-9(2) 5$.

Sukhdeep, B., Akansha, P., Anindita, R., Arpan, D. 2016. Blind Navigation System. 3rd International Conference on Recent Trends in Engineering Science and Management (ICRTESM), 780-785.

Ganguli, A., Pushp, D., Chaudhary, A. 2016. Electronic Travel Aids ETA for Blind Assistance. International Journal of Engineering Science and Computing (IJESC), 2672-2675.

Gassert, R., and Team. 2013. White Cane With Integrated Electronic Travel AD Using 3D TOF Sensor. The United States Patent Application Publication, US2013/0220392A1.

Le, M., Phung, S. Lam. \& Bouzerdoum, A. 2012. Pedestrian lane detection for assistive navigation of blind people. 21st International Conference on Pattern Recognition (ICPR), 2594-2597.

Le, M., Phung, S., Bouzerdoum, A. 2014. Pedestrian Lane Detection in Unstructured Environments for Assistive Navigation. International Conference on Digital Image Computing: Techniques and Applications (DICTA), 1-8.

Mocanu, I., Clapton, C. 2018. Multimodal Convolutional Neural Network for Object Detection Using RGB-D Images. 41st International Conference on Telecommunications and Signal Processing (TSP), Athens, $1-5$.

Rao, K., Jyothi, K., Mahamood, S. 2015. Secure Navigation for the Blind People by Using RFID. International Journal Of Innovative Research In Electrical Electronics Instrumentation and Control Engineering (IJIREEICE ), 132-135.

Setiadi, B., Supriyadi, T. 2017. Development Of Visual Sensory Aids Using Embedded System for Blind Person. Proceeding the-6 International Seminar ASAIS- Politeknik Negeri Jakarta, 1-6.

Setiadi, B., Supriyadi, T., Nugroho, H. 2018. Development Of Navigation Visual Sensory Aids For Blind Persons Using Camera. Proceeding ICSTR-Jakarta Universitas Al Azhar Jakarta.

Wahab, M., Talib, A., Kadir, H., Johari, A., Noraziah, A., Sidek, M., Mutalib, A. 2011. Smart cane: assistive cane for visually impaired people. International Journal of Computer Science Issues (IJCSI), 21-27.

Wang, S., Tian, Y. 2012. Detecting Stairs and Pedestrian Crosswalks for the Blind by RGBD Camera. IEEE International Conference on Bioinformatics and Biomedicine Workshops, Philadelphia, 732739.

Wenlong, S., Yupeng, C., Xuefu, C. 2016. The Design of a Guide Device with Multi-Function to Aid Travel for Blind Person. International Journal of Smart Home (IJSH), 77-86.

Ye, C., Qian, X. 2018. 3-D Object Recognition of a Robotic Navigation Aid for the Visually Impaired. IEEE Transactions on Neural Systems and Rehabilitation Engineering, 441-450.

Yi, C., Tian, Y., Arditi, A. 2014. Portable Camera-Based Assistive Text and Product Label Reading From Hand-Held Objects for Blind Persons. IEEE/ASME Transactions on Mechatronics, 808-817. 